\title{
Experimental Measurement of the Generalized Stokes Parameters of a Radially Polarized Random Electromagnetic Beam
}

\author{
Yongxin Liu' ${ }^{*}$, Songjie Luo ${ }^{1}$, Jixiong Pu${ }^{1}$, Zenghui Gao ${ }^{2}$ \\ ${ }^{1}$ Fujian Provincial Key Laboratory of Light Propagation and Transformation, Huaqiao University, Xiamen, China \\ ${ }^{2}$ Key Laboratory of Computational Physics, Yibin University, Yibin, China \\ Email: 22254208@qq.com, songjieluo@163.com, jixiong@hqu.edu.cn
}

Received 25 May 2016; accepted 14 June 2016; published 17 June 2016

Copyright (C) 2016 by authors and Scientific Research Publishing Inc.

This work is licensed under the Creative Commons Attribution International License (CC BY).

http://creativecommons.org/licenses/by/4.0/

(c) (i) Open Access

\begin{abstract}
Utilizing the Young's double slits and Mach-Zehnder interferometer, we proposed an experimental method to measure the generalized Stokes parameters of a radially polarized random electromagnetic beam. After the partially coherent beam propagating through the Young's double slits, the interference fringe is obtained by the help of a Mach-Zehnder interferometer consisting of apertures, quarter-wave plates and polarizers. The electric cross-spectral density matrix is detected by the coherence degree of interference fringe and the density of each single slit. The generalized Stokes parameters can be obtained from the electric cross-spectral density matrix. This experiment measures the generalized Stokes parameters of the random electromagnetic beam successfully. The results show that the spectral degree of coherence for copolarized cases $(x x$ and $y y)$ is similar with that for cross-polaried cases $(x y$ and $y x)$ for the radially polarized random electromagnetic beam. This method will help us determine the change of the polarization and coherence of the light in propagation by detecting the change of the generalized Stokes parameters.
\end{abstract}

\section{Keywords}

Generalized Stokes Parameters, Electric Cross-Spectral Density Matrix, Young's Double Slits, Mach-Zehnder Interferometer, Radially Polarized Stochastic Electromagnetic Beam

\section{Introduction}

Coherence and polarization of the electromagnetic beams are always considered as two unrelated subjects to in-

${ }^{*}$ Corresponding author.

How to cite this paper: Liu, Y.X., Luo, S.J., Pu, J.X. and Gao, Z.H. (2016) Experimental Measurement of the Generalized Stokes Parameters of a Radially Polarized Random Electromagnetic Beam. Journal of Electromagnetic Analysis and Applications, 8, 109-114. http://dx.doi.org/10.4236/jemaa.2016.86011 
vestigate and discuss. However, both of them have the same physical phenomenon, that is, the correlation between the fluctuations of the electric field. Coherence is caused by the fluctuation of the electric field of two or more spatial points. Polarization is related to the fluctuation of the component of the electric field of the same spatial point.

In 2003, Emil Wolf developed the unified theory of coherence and polarization of a random electromagnetic beam, which illuminated an intimate relationship existing between the coherence and polarization of electromagnetic beams [1]. Then, how to use the cross spectral density matrix of the electric field to calculate the degree of polarization, the degree of coherence and the spectrum of a random electromagnetic beam in the propagation is analyzed theoretically [2]-[4]. And next, Emil Wolf presented a method of how the four elements of the cross spectral density matrix may be determined from measurements of spectra in the Young's interference experiment in which polarizers and rotators are placed in front of the pinholes [5].

Recently, the generalized Stokes parameter, which is a new extension associated with two spatial points, also depends on the cross spectral density matrix of the electric field [6]. Therefore, the generalized Stokes parameter can be used to study the coherence and polarization of the electromagnetic beam.

In 2008, Kamdpal H. proposed an experimental method of measuring the cross spectral density matrix and the generalized Stokes parameters of the laser beam [7], but it is still difficult to be realized in the ordinary laboratory, because of the demanding experimental structures. In 2009, C. Kamdpal H. also pointed out another method which can determine the generalized Stokes parameters of the beam by directly and experimentally measuring the ordinary Stokes parameters, but the use of the beam is completely coherent laser beam [8].

Meanwhile, the radially polarized beam can be focused to generate a strong longitudinal and non-propagating electric field at the focal plane through a high numerical aperture objective [9] [10]. Due to this unique feature, the radially polarized beam has attracted significant interest [11] [12]. In this paper, we will investigate the generalized Stokes parameters of a radially polarized random electromagnetic beam experimentally. We propose an experimental method utilizing a double-slit aperture and a Mach-Zehnder interferometer to measure the cross spectral density matrix and generalized Stokes parameters of a radially polarized random electromagnetic beam. A rotating ground glass and a radial polarization converter are placed in front of the double-slit aperture to produce a radially polarized partially coherent beam, and the polarizers and half wave plates in the Mach-Zehnder interferometer are used to take measurements of the four elements of the cross spectral density matrix.

\section{Formulation}

Let us consider a quasi monochromatic light propagating along the $Z$ direction. The $2 \times 2$ cross-spectral density matrix can be given by:

$$
W\left(r_{1}, r_{2}, \omega\right)=\left[\begin{array}{ll}
W_{x x}\left(r_{1}, r_{2}, \omega\right) & W_{x y}\left(r_{1}, r_{2}, \omega\right) \\
W_{y x}\left(r_{1}, r_{2}, \omega\right) & W_{y y}\left(r_{1}, r_{2}, \omega\right)
\end{array}\right]
$$

where the $r_{1}$ and $r_{2}$ are the coordinate vectors on the plane $Z$, and

$$
W_{i j}\left(r_{1}, r_{2}, \omega\right)=\left\langle E_{i}^{*}\left(r_{1}, \omega\right) E_{j}\left(r_{2}, \omega\right)\right\rangle, i=x, y, j=x, y,
$$

where the asterisk denotes the complex conjugate and the angular brackets denote the ensemble average. $E_{i}$ and $E_{j}$ are components of the electric field.

The spectral degree of coherence of the electric field of the beam at the points $r_{1}$ and $r_{2}$ can be expressed as [5]:

$$
\eta\left(r_{1}, r_{2}, \omega\right)=\frac{\operatorname{Tr} W\left(r_{1}, r_{2}, \omega\right)}{\sqrt{\operatorname{Tr} W\left(r_{1}, r_{1}, \omega\right)} \sqrt{\operatorname{Tr} W\left(r_{2}, r_{2}, \omega\right)}}
$$

where, $\operatorname{Tr}$ is the matrix trace.

The spectral density can be given by:

$$
\begin{aligned}
& S\left(r_{1}, \omega\right)=\operatorname{Tr} W\left(r_{1}, r_{1}, \omega\right) \\
& S\left(r_{2}, \omega\right)=\operatorname{Tr} W\left(r_{2}, r_{2}, \omega\right)
\end{aligned}
$$

Then, Equation (3) can be simplified as: 


$$
\operatorname{TrW}\left(r_{1}, r_{2}, \omega\right)=\sqrt{S\left(r_{1}, \omega\right)} \sqrt{S\left(r_{2}, \omega\right)} \eta\left(r_{1}, r_{2}, \omega\right)
$$

According to Ref. [5], using the Young's double slits, all the four elements of the electric cross-spectral density matrix are determined as follows:

$$
\begin{aligned}
& W_{x x}\left(r_{1}, r_{2}, \omega\right)=\sqrt{S_{x}\left(r_{x}, \omega\right)} \sqrt{S_{x}\left(r_{x}, \omega\right)} \eta_{x x}\left(r_{1}, r_{2}, \omega\right) \\
& W_{y y}\left(r_{1}, r_{2}, \omega\right)=\sqrt{S_{y}\left(r_{1}, \omega\right)} \sqrt{S_{y}\left(r_{2}, \omega\right)} \eta_{y y}\left(r_{1}, r_{2}, \omega\right) \\
& W_{x y}\left(r_{1}, r_{2}, \omega\right)=\sqrt{S_{x}\left(r_{1}, \omega\right)} \sqrt{S_{y}\left(r_{2}, \omega\right)} \eta_{x y}\left(r_{1}, r_{2}, \omega\right) \\
& W_{y x}\left(r_{1}, r_{2}, \omega\right)=\sqrt{S_{y}\left(r_{1}, \omega\right)} \sqrt{S_{x}\left(r_{2}, \omega\right)} \eta_{y x}\left(r_{1}, r_{2}, \omega\right)
\end{aligned}
$$

The generalized stokes parameters can be given by:

$$
\begin{aligned}
& S_{0}\left(r_{1}, r_{2}, \omega\right)=W_{x x}\left(r_{1}, r_{2}, \omega\right)+W_{y y}\left(r_{1}, r_{2}, \omega\right) \\
& S_{1}\left(r_{1}, r_{2}, \omega\right)=W_{x x}\left(r_{1}, r_{2}, \omega\right)-W_{y y}\left(r_{1}, r_{2}, \omega\right) \\
& S_{2}\left(r_{1}, r_{2}, \omega\right)=W_{x y}\left(r_{1}, r_{2}, \omega\right)+W_{y x}\left(r_{1}, r_{2}, \omega\right) \\
& S_{3}\left(r_{1}, r_{2}, \omega\right)=i\left[W_{y x}\left(r_{1}, r_{2}, \omega\right)-W_{x y}\left(r_{1}, r_{2}, \omega\right)\right]
\end{aligned}
$$

In the coming section, we will present a method to measure the four elements of the electric cross-spectral density matrix and the generalized stokes parameters of a beam on the basis of Equation (6) and Equation (7).

\section{Results and Discussions}

The schematic of the experimental setup is shown in Figure 1. A linear polarized helium-neon laser is incident over a radially polarization converter to produce the radially polarized beam. Then, the beam is expanded by an expanding system which containing a rotating ground glass in the focus to reduce the coherence of the light. The newly generated radially polarized partially coherent beam passes through a Young's double slit and a MachZehnder interferometer consisting of apertures, quarter-wave plates and polarizers. The interference fringes can be obtained in the observation plane as shown in Figure 2.

To calculate the $W_{x x}\left(r_{1}, r_{2}, \omega\right)$ component, it is necessary to keep the polarization of two polarizers in $x$ axis direction. And the direction of optic axis of two half wave plates should be the same with $x$ axis (to ensure the same optical loss of different condition). This allows only the $x$ component of the electric field to pass through.

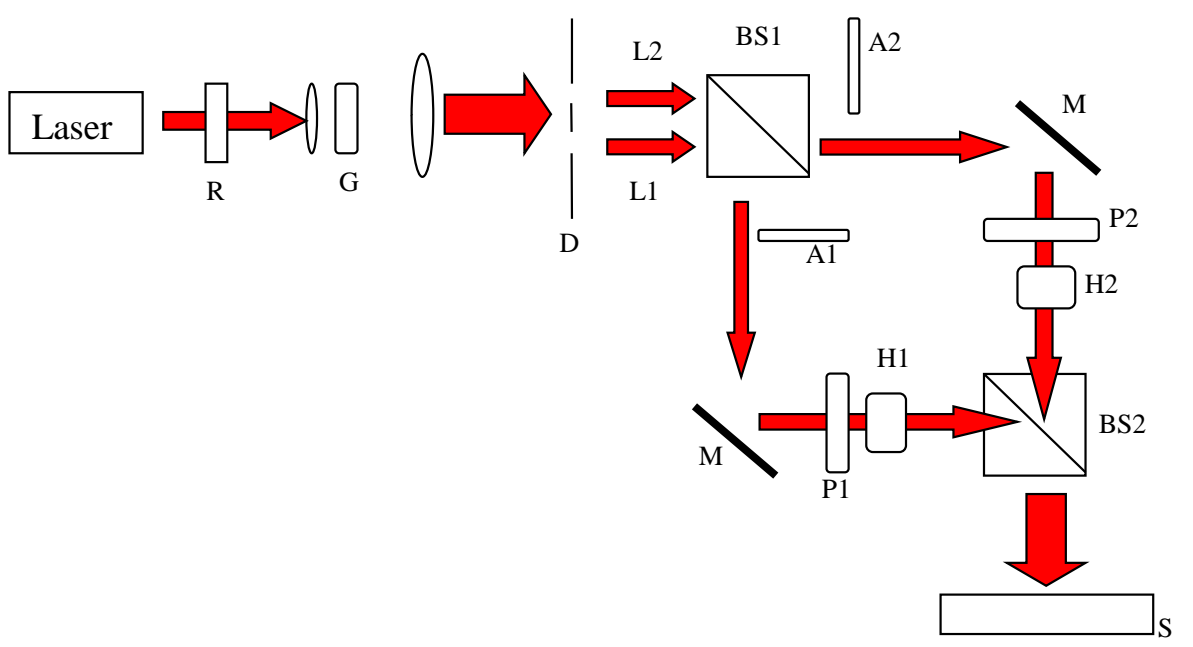

Figure 1. The schematics of the experimental setup. $\mathrm{R}$ is a radial polarization converter; $\mathrm{G}$ is a ground glass; $\mathrm{D}$ is a Young's double slits with slit width $0.1 \mathrm{~mm}$ and slit separation $1.6 \mathrm{~mm}$; $\mathrm{L}_{1}$ and $\mathrm{L}_{2}$ are beams coming from the Young's double slits; $\mathrm{BS}$ is a beam splitter; $\mathrm{A}$ is an aperture; $\mathrm{P}$ is a polarizing film, $\mathrm{H}$ is a half wave plate; $\mathrm{S}$ is a spectrometer. 


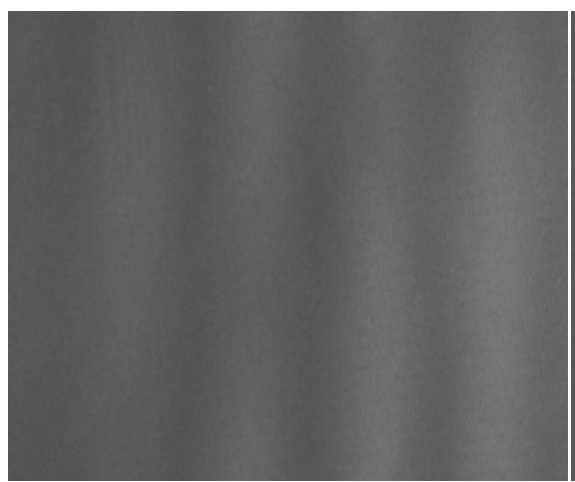

(a)

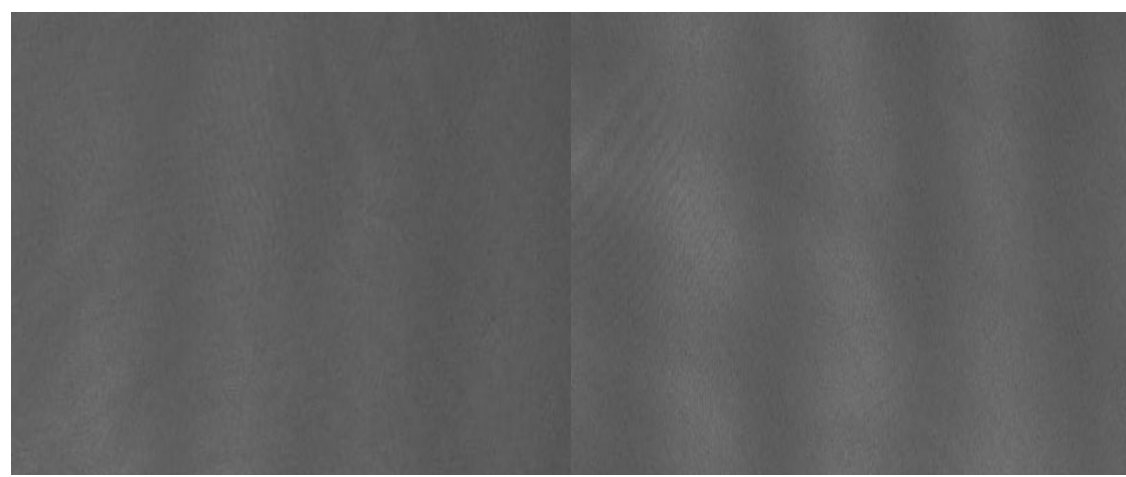

(c)

(d)

Figure 2. The figure of interference fringe at the plate $S$. (a) $x x$ polarization; (b) yy polarization; (c) $x y$ polarization; (d) $y x$ polarization.

The aperture $A_{1}$ only allows $L_{2}$ to pass and $A_{2}$ allows $L_{1}$ to pass. Then, the interference fringes of the doubleslits are obtained on the observation plane. The maximum and minimum values of the spectral density of the central fringe are recorded, and the spectral densities due to each individual slits are also recorded at the same point by a spectrometer. To get the $W_{y y}\left(r_{1}, r_{2}, \omega\right)$ component, the polarizers and half wave plates should be rotated clockwise by $90^{\circ}$ (this change the direction of polarization to $y$ axis) and repeat the similar steps.

The $W_{x y}\left(r_{1}, r_{2}, \omega\right)$ component can be obtained when the directions of polarization of two polarizers are perpendicular. The fast axis of the half wave plate $\left(\mathrm{H}_{1}\right)$ which is followed by the polarizer $\left(\mathrm{P}_{1}\right)$ with $x$ direction of polarization should keep the $45^{\circ}$ with the $x$ axis. Therefore, the polarization of incident light will change from the $x$ direction to $y$ direction. At this time, the interference fringes appear when the polarization direction of the polarizer $\left(\mathrm{P}_{2}\right)$ and the half wave plate $\left(\mathrm{H}_{2}\right)$ fast axis are kept along $y$ axis in another arm. The maximum and minimum values around the central fringe and spectral densities of each single slit in the same position should be recorded. The $W_{y x}\left(r_{1}, r_{2}, \omega\right)$ component can be gotten when the direction of polarization of polarizer $\left(\mathrm{P}_{1}\right)$ and the fast axis of the half wave plate $\left(\mathrm{H}_{1}\right)$ are kept the same with $y$ axis. Meanwhile, the polarizer $\left(\mathrm{P}_{2}\right)$ is along the $x$ axis and the half wave plate $\left(\mathrm{H}_{2}\right)$, having optics axis at $45^{\circ}$ with $x$ axis, works as a $90^{\circ}$ polarization rotator in another arm. Similar measurements for both the slits as well as the individual slits are taken.

As the Figure 2 shows, the visibility of interference fringe of radially polarized random electromagnetic beam due to different components is similar.

With the help of values from spectrometer, the spectral degree of coherence can be calculated by the equation:

$$
\eta=\frac{S_{\max }-S_{\min }}{S_{\max }+S_{\min }}
$$

where the $S_{\max }$ and $S_{\min }$ are the maximum and minimum values of spectral intensity around the central fringe when both the slits are open.

The coherences of the four conditions are given by Table 1. Utilizing the spectral intensity of single slit, the electric cross-spectral density matrix can be calculated. After repeated measurement experiments four times, the 
average values and the deviation have been worked out. According to Equations (7a)-(7d), the generalized Stokes parameters can be obtained immediately as shown in Table 2.

According to the Table 1, the spectral coherence of the same polarized direction component ( $x x$ and $y y)$ of the radially polarized light is similar with that for different polarized direction component ( $x y$ and $y x$ ). It is because that there is almost the same correlation between the two orthogonal components or the parallel components of the radially polarized partially coherent beam.

For comparison, the interference fringes of a randomly polarized partially coherent laser have been experimentally obtained as shown in Figure 3. From the Figure 3, we can see that the visibility of interference fringes

Table 1. Components of the spectral degree of coherence and the cross-spectral density matrix obtained from the laser light.

\begin{tabular}{ccc}
\hline Polarization & Degree of coherence $\eta\left(r_{1}, r_{2}, \omega\right)$ & Cross-spectral density matrix $W\left(r_{1}, r_{2}, \omega\right)$ \\
\hline$x x$ & $0.33 \pm 0.02$ & $52.24 \pm 4.71$ \\
$x y$ & $0.26 \pm 0.03$ & $40.04 \pm 4.62$ \\
$y x$ & $0.32 \pm 0.03$ & $51.79 \pm 4.86$ \\
$y y$ & $0.37 \pm 0.04$ & $68.31 \pm 7.38$ \\
\hline
\end{tabular}

Table 2. Generalized stokes parameters for a pair of points in the random electromagnetic beam.

\begin{tabular}{cc} 
Generalized stokes parameters & Value \\
\hline$S_{0}\left(r_{1}, r_{2}, \omega\right)$ & $120.55 \pm 6.37$ \\
$S_{1}\left(r_{1}, r_{2}, \omega\right)$ & $16.07 \pm 2.90$ \\
$S_{2}\left(r_{1}, r_{2}, \omega\right)$ & $91.83 \pm 2.33$ \\
$S_{3}\left(r_{1}, r_{2}, \omega\right)$ & $(16.52 \pm 1.41) i$ \\
\hline
\end{tabular}

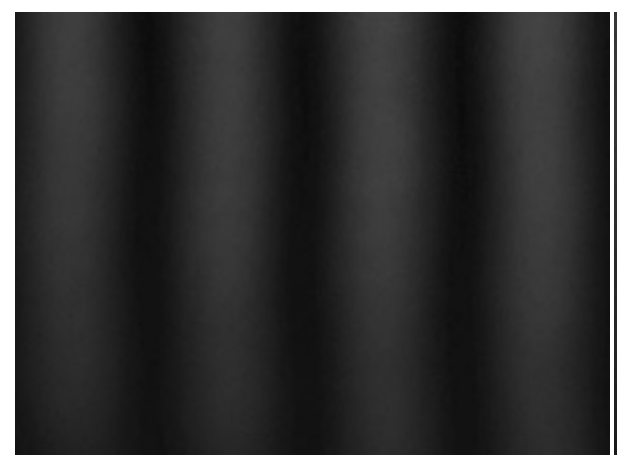

(a)

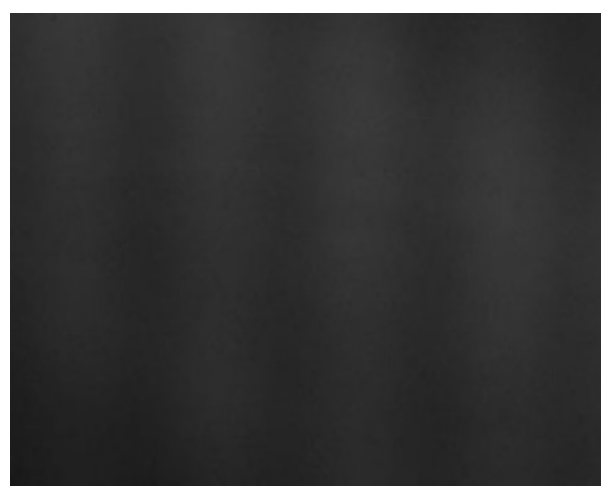

(c)

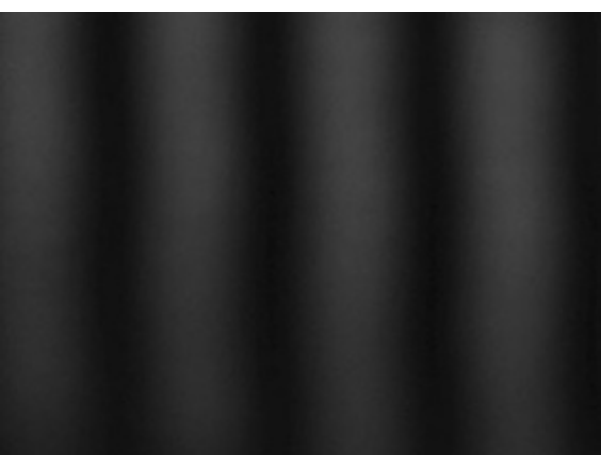

(b)

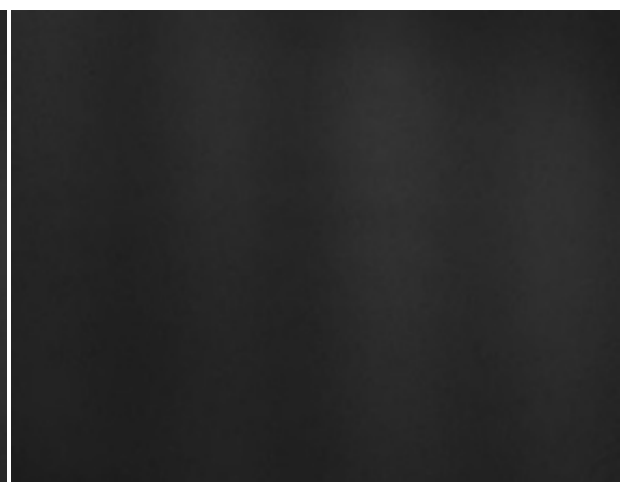

(d)

Figure 3. The interference distribution of a randomly polarized partially coherent laser beam at the plate $S$. (a) $x x$ polarization; (b) yy polarization; (c) xy polarization; (d) yx polarization. 
for $x y$ components and $y x$ components is less than that for $x x$ components and $y y$ components. It is due to the fact that there is very little correlation between the two orthogonal components of a randomly polarized light.

\section{Conclusion}

In conclusion, we have experimentally measured the four elements of the electric cross-spectral density matrix of a radially polarized partially coherent laser by the simple equipment. The generalized Stocks parameters can be computed by the cross-spectral density matrix. The method measures the coherence of two different points of the radially polarized laser. The result shows that there is almost the same correlation between the two orthogonal components or the parallel components of the radially polarized partially coherent beam, which is different from the randomly polarized partially coherent laser. The generalized Stocks parameters help us to study the properties of coherence and polarization of the radially polarized stochastic electromagnetic beams in propagation.

\section{Acknowledgements}

This research was supported by the National Natural Science Foundation of China (Nos. 61275203; 61505059; 61515070), the High level talent research of Huaqiao University (12BS231), and the Natural Science Foundation of Fujian Province of China (No. 2014J05007).

\section{References}

[1] Wolf, E. (2003) Unified Theory of Coherence and Polarization of Random Electromagnetic Beams. Physics Letters A, 312, 263-267. http://dx.doi.org/10.1016/S0375-9601(03)00684-4

[2] Wolf, E. (2003) Correlation-Induced Changes in the Degree of Polarization, the Degree of Coherence, and the Spectrum of Random Electromagnetic Beams on Propagation. Optics Letters, 28, 1078-1080. http://dx.doi.org/10.1364/OL.28.001078

[3] Agrawal, G.P. and Wolf, E. (2009) Propagation-Induced Polarization Changes Impartially Coherent Optical Beams. Optical Society of America A, 17, 2019. http://dx.doi.org/10.1364/JOSAA.17.002019

[4] Wolf, E. (2007) Introduction to Theory of Coherence and Polarization of Light. Cambridge University Press, Cambridge.

[5] Roychowdlhury, H. and Wolf, E. (2003) Determination of the Electric Cross-Spectral Density Matrix of a Random Electromagnetic Beam. Optics Communications, 226, 57-60. http://dx.doi.org/10.1016/j.optcom.2003.07.054

[6] Korotkova, O. and Wolf, E. (2005) Generalized Stokes Parameters of Random Electromagnetic Beams. Optics Letters, 30, 198-200. http://dx.doi.org/10.1364/OL.30.000198

[7] Kanseri, B. and Kamdpal, H.C. (2008) Experimental Determination of Electric Cross-Spectral Density Matrix and Generalized Stokes Parameters for a Laser Beam. Optics Letters, 33, 2410-2412. http://dx.doi.org/10.1364/OL.33.002410

[8] Kanseri, B., Rath, S. and Kandpal, H.C. (2009) Direct Determination of the Generalized Stokes Parameters from the Usual Stokes Parameters. Optics Letters, 34,719-721. http://dx.doi.org/10.1364/OL.34.000719

[9] Dorn, R., Quabis, S. and Leuchs, G. (2003) Sharper Focus for a Radially Polarized Light Beam. Physical Review Letters, 91, 233901. http://dx.doi.org/10.1103/PhysRevLett.91.233901

[10] Lin, H., Jia, B.H. and Gu, M. (2011) Generation of an Axially Super-Resolved Quasi-Spherical Focal Spot Using an Amplitude-Modulated Radially Polarized Beam. Optics Letters, 36, 2471-2473. http://dx.doi.org/10.1364/OL.36.002471

[11] Zhang, Y., Ding, B. and Taikei, S. (2010) Trapping Two Types of Particles Using a Double-Ring-Shaped Radially Polarized Beam. Physical Review A, 81, 023831. http://dx.doi.org/10.1103/PhysRevA.81.023831

[12] Yan, S. and Yao, B. (2007) Radiation Forces of a Highly Focused Radially Polarized Beam on Spherical Particles. Physical Review A, 76, 053836. http://dx.doi.org/10.1103/PhysRevA.76.053836 


\section{Submit or recommend next manuscript to SCIRP and we will provide best service for you:}

Accepting pre-submission inquiries through Email, Facebook, Linkedin, Twitter, etc A wide selection of journals (inclusive of 9 subjects, more than 200 journals)

Providing a 24-hour high-quality service

User-friendly online submission system

Fair and swift peer-review system

Efficient typesetting and proofreading procedure

Display of the result of downloads and visits, as well as the number of cited articles

Maximum dissemination of your research work

Submit your manuscript at: http://papersubmission.scirp.org/ 